\title{
Giant Cavernous Malformation Presenting as a Large Cystic Intracranial Mass with an Enhancing Mural Nodule: A Case Report and Review of the Literature
}

\section{Peter Amenta, MD, James J. Evans, MD}

Department of Neurological Surgery, Thomas Jefferson University, Philadelphia, PA

\section{Abstract}

\section{Background}

Cavernous malformations are relatively rare vascular anomalies which typically follow a benign clinical course. The vast majority of these lesions are small and only become clinically significant when they induce seizures or hemorrhage. Rarely, cavernous malformations may grow to large sizes with-
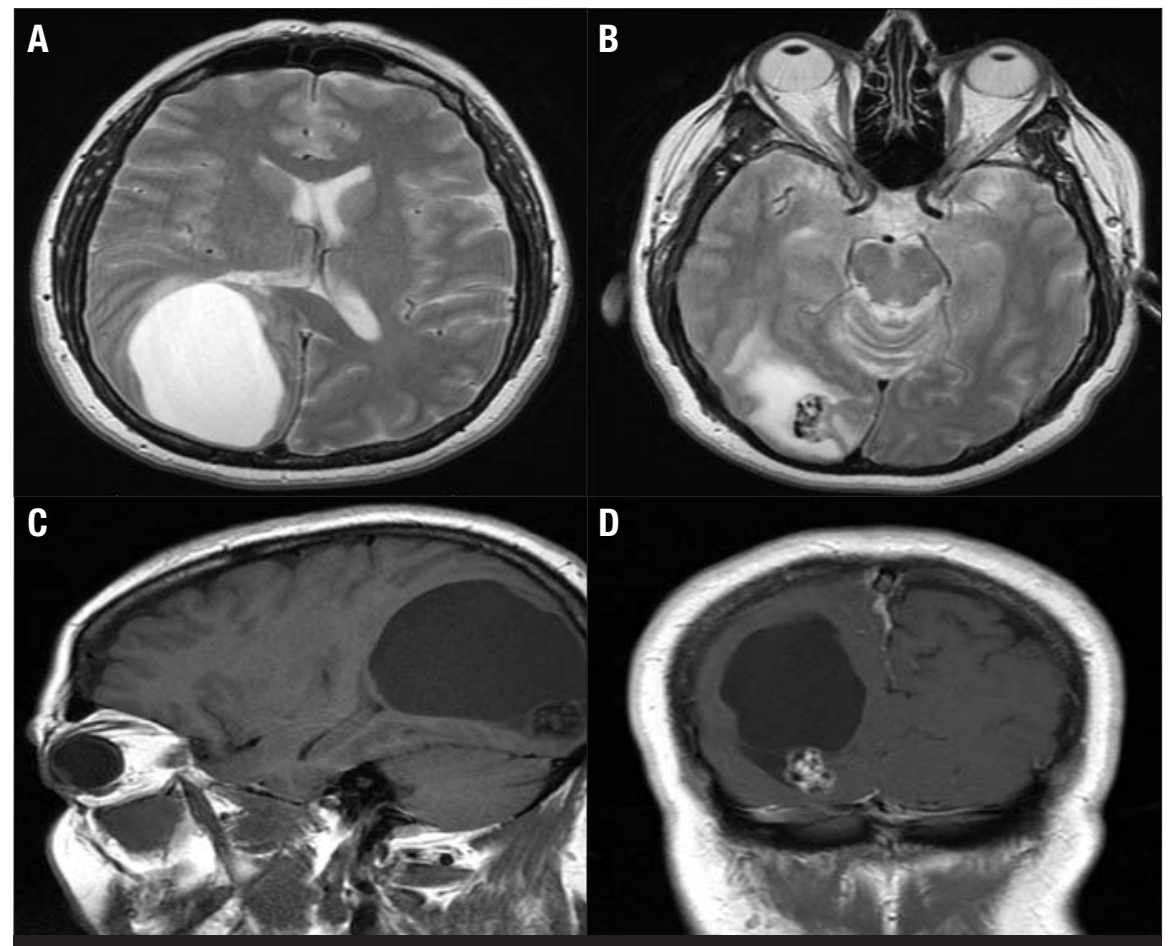

Figure 1

(A) Preoperative MRI. T2-weighted axial MRI shows a large occipital hyperintense cystic lesion with local mass effect and minimal surrounding edema. (B) A heterogeneous mural nodule is exhibited with hypodensities suggestive of calcification or prominent intralesional flow voids. (C) T1-weighted non-contrast sagittal image demonstrates cystic mass and relatively hypodense mural nodule. (D) T1-weighted contrast-enhanced coronal image reveals heterogeneous enhancement of the mural nodule and a lack of enhancement of the cystic component. out a clinically significant acute hemorrhage, thus resulting in a presentation consistent with a large intracranial mass lesion. These giant cavernous malformations present a diagnostic challenge, as CT and MRI are frequently unable to distinguish them from neoplastic pathology.

\section{Case Description}

We present the case of an adult female who presented with a slowly progressing left homonymous hemianopsia. MRI revealed a large right occipital cystic mass with an enhancing mural nodule and possible intralesional flow voids. Initial diagnosis based on imaging was consistent with hemangioblastoma. Following a negative preoperative angiogram and surgical resection, final pathology determined the lesion to be a giant cavernous malformation.

\section{Conclusion}

This case demonstrates the limitations of current imaging techniques and highlights the variability in the natural history, clinical presentation, and imaging characteristics of these rare lesions.

\section{Key Words}

Giant cavernous malformation, intracranial mass lesion, vascular malformation

\section{Case Report}

The patient is a 52-year-old female with a past medical history significant for ocular migraines since childhood. She presented to her family practitioner with complaints of progressively diminishing visual acuity in the left visual field over the course of two months. Neurological examination revealed a dense left homonymous hemianopsia. MRI demonstrated a large rightsided occipital cyst and associated $1.9 \times 2 \times 2.2$ $\mathrm{cm}$ heterogeneously enhancing nodular mass with possible heavy calcium deposition or flow-voids. The cyst surrounding the nodule did not enhance or produce midline shift, but did cause local mass effect and compression of the right occipital horn. There was minimal surrounding edema as evidenced by the $\mathrm{T} 2$ and FLAIR sequences. Imaging was believed to be 
consistent with a hemangioblastoma (Figure 1). As a result, prior to surgical resection, a cerebral angiogram was performed to evaluate for the presence of arterial feeding vessels amenable to embolization. Angiographic evaluation was negative for arterial pedicles or associated vascular malformation.

The patient was positioned prone and a large occipital craniotomy was performed via a right-sided paramedian sigmoid incision. A small cortisectomy immediately expressed a large volume of xanthochromic cystic fluid, thus relaxing the surrounding parenchyma. The remaining cavity was lined with a translucent membrane and an inferiorly based firm, calcified mass was found to be adherent to the surrounding tissue. The mass was resected en bloc with minimal hemorrhage (Figure 2).

Frozen pathology revealed a heavily calcified lesion, but could not differentiate between hemangioblastoma and cavernous malformation. Final pathologic examination revealed multiple areas of back-to-back vascular channels composed of fibrotic, amuscular walls that stained positive for the endothelial cell markers CD34 and CD31. The remaining tissue was consistent with reactive gliosis, confirming the diagnosis of cavernous malformation.

The patient awoke with a persistent left homonymous hemianopsia which showed minimal improvement on postoperative day number two. At one month follow-up, the patient continued to report ocular migraines, however, formal visual field testing revealed full visual fields.

\section{Discussion}

Cavernous malformations are relatively rare vascular malformations with a reported incidence of 0.4 to $0.9 \%$ based on autopsy and magnetic resonance imaging (MRI) series. ${ }^{1}$ These vascular anomalies are composed of abnormal cavernous endothelial-lined spaces lacking smooth muscle and intervening neural tissue. Within these channels, static blood flow results in thrombosis, hematoma organization, and eventual calcification. Cavernous malformations most commonly occur sporadically and exist as solitary lesions. ${ }^{2-4}$ However, the familial form of the disease, which results in the formation of multiple lesions, has been localized to mutations in several chromosomes. ${ }^{5}$ On average, individual lesions exist over a large spectrum of sizes, ranging from a few millimeters to multiple centimeters. Rarely, cavernous malformations may grow to a large size, with a diameter of $6 \mathrm{~cm}$ defining the lesion as a giant cavernous malformation (GCM). ${ }^{6}$ These larger masses represent a

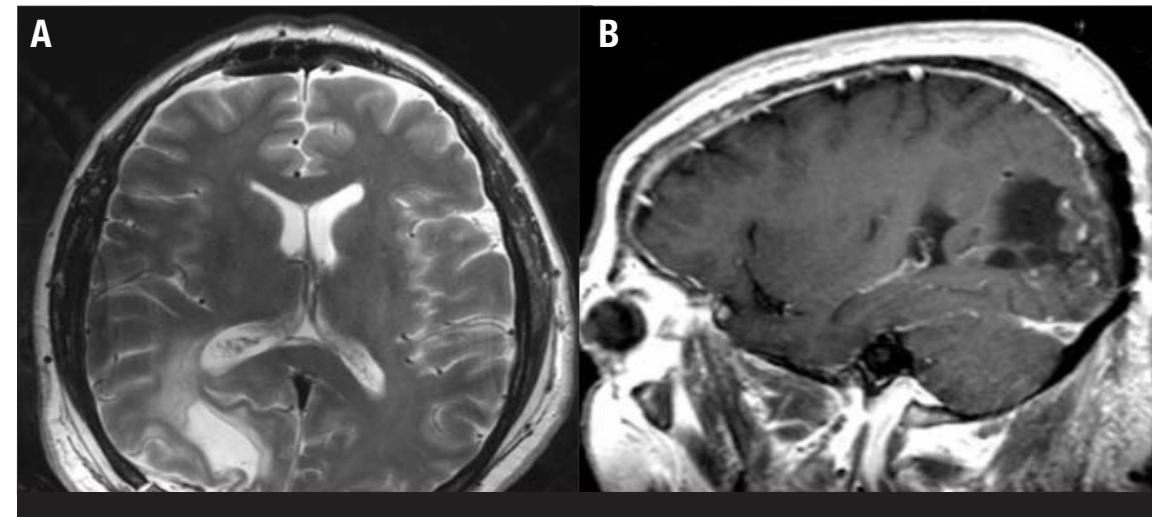

Figure 2

(A) Postoperative MRI. T2-weighted axial image shows decompressed occipital cyst and decompressed right occipital horn. (B) T1-weighted contrast axial image reveals no residual mural nodule.

distinct disease entity that may often mimic tumor pathology in imaging characteristics, pattern of growth, and clinical presentation. ${ }^{7}$

Modern imaging techniques have contributed to a greater understanding of the natural history of cavernous malformations. Cavernous malformations have been traditionally considered congenital, yet de novo formation of new lesions has been demonstrated in the familial form of the disease and following whole brain and stereotactic radiation. ${ }^{8}$ Additionally, serial imaging has shown growth in the size of cavernous malformations over time, including enlargement of the nodular mass, and less commonly, development of a cystic component. Cystic enlargement is believed to result from intralesional hemorrhage, breakdown and accumulation of blood products, and encapsulation ${ }^{9}$

\section{Imaging Characteristics}

Typical cavernous malformations are often difficult to appreciate on standard CT, however, when identifiable, they appear as hyperdense parenchymal lesions. T2-weighted and gradient echo MRI sequences reveal hemosiderin deposition and hematomas in various states of maturation, resulting in the nearly pathognomonic image of a circumscribed, heterogeneous mass with an irregular "popcorn" appearance., 910 Lesional enhancement is rare, and when present, is often faint. ${ }^{11}$

Based on the existing literature, the radiographic appearance of GCMs is highly variable and consists of completely solid, primarily cystic, or heterogeneous masses composed of both components. ${ }^{12}$ The presence of contrast enhancement is also inconsistent, ranging from nonexistent to intensely enhancing. A number of case reports describe a tumefactive appearance on MRI, as the lesions demonstrate an apparent infiltrative pattern and significant perilesional edema. ${ }^{1,6,13,14}$ These masses may mimic the appearance of high-grade glial neoplasms or hemorrhagic tumors, such as, oligodendrogliomas and metastases. Further complicating the differential diagnosis are largely cystic masses without surrounding edema or an infiltrative appearance. In the pediatric population, GCMs with a cystic component and an apparent mural nodule have been mistaken for pilocytic astrocytomas. ${ }^{12}$ We present a similar GCM in an adult with long-standing ocular migraines and imaging consistent with a hemangioblastoma. Furthermore, the degree of calcification encountered during resection rendered the mass unidentifiable on gross examination and at the time of frozen section analysis.

\section{Clinical Presentation}

The clinical presentation of a GCM exists over a broad spectrum, ranging from headaches to catastrophic life-threatening hemorrhage. A significant number of GCMs present classically, with a seizure, acute-onset of a severe headache, or new focal neurologic deficit. ${ }^{15}$ Large, slowly-expanding lesions may present with manifestations of increased intracranial pressure due to obstructive hydrocephalus or the result of a significant space-occupying mass. The insidious onset of a progressive neurologic deficit, as was seen in our case, has been reported in the literature and is the result of slow lesional growth in the absence of a significant hemorrhage. ${ }^{9}$ Of greatest importance is the realization that hemorrhage of a GCM must be included in the differential diagnosis of non-traumatic 
spontaneous intracerebral hemorrhage. These patients may present with acute hemiparesis, seizures, and cerebral herniation syndromes.

\section{Treatment}

The vast majority of cavernomas do not require surgical resection due to their relatively benign clinical course. When indicated, current neurosurgical management of cavernous malformations, regardless of size, consists of image-guided complete surgical resection if possible. Standard indications for surgical resection include recurrent hemorrhage, progressive neurologic deterioration, and medically refractory epilepsy. ${ }^{16}$ For cavernous malformations in which resection is associated with an unacceptably high risk, such as location in eloquent parenchyma, stereotactic radiosurgery has been attempted with reports of varying success and a possible increased risk of subsequent hemorrhage. Multiple case reports of favorable surgical outcomes with resection of GCMs are found throughout the literature. ${ }^{17-19}$

\section{Conclusion}

We report the subacute presentation of a GCM in a patient with a slowly progressive neurologic deficit. This case provides an example of the limitations of current imaging techniques and serves to broaden the differential diagnosis for large cystic intracranial masses. Furthermore, the variability in the natural history, clinical presentation, and imaging characteristics of GCMs is highlighted.

\section{References}

1. Van Lindert EJ, T. T., Grotenhuis JA, Wesseling P. Giant cavernous hemangiomas: report of three cases. Neurosurg Rev 30, 83-92 (2007).

2. Kesava PP, T. P. MR angiography of vascular malformations. Neuroimaging Clin N Am 8, 349-370 (1998).

3. Kitamura K, F. M., Oka K, Matsushima T, Hasuo K, et al. Hemangiomas of the central nervous system in Japan: An epidemiological and clinicopathological study with special reference to venous and cavernous malformations. Neurosurg Rev 9, 221-231 (1986).

4. Avci E, O. A., Baba F, Karabag H, Cakir A. Huge cavernoma with massive intracerebral hemorrhage in a child. Turkish Neurosurgery 17, 23-26 (2007).

5. Craig HD, G. M., Cepeda O, Johnson EW, et al. Multilocus linkage identifies two new loci for a Mendelian form of stroke, cerebral cavernous malformation, at $7 \mathrm{p} 15-13$ and 3q25.2-27. Hum Mol Genet 7, 1851-1858 (1998)

6. Lawton MT, V. G., Quinones-Hinojosa A, et al. Giant infiltrative cavernous malformation: clinical presentation, intervention, and genetic analysis: case report. Neurosurgery 55, 988-995 (2004)

7. Steinberg GK, M. M. Lesions mimicking cavernous malformations. Vol. 151-162 (AANS Publications Committee, 1993).

8. Larson JJ, B. W., Bove KE, Crone KR, Tew JM Jr. Formation of intracerebral cavernous malformations after radiation treatment for central nervous system neoplasia in children. $J$ Neurosurg 88, 51-56 (1998).
9. Chicani CF, M. N., Tamargo RJ. J Neuroophthalmol. Giant cavernous malformation of the occipital lobe 23, 151-153 (2003).

10. Simard JM, G.-B. F., Ballinger WE, Mickle JP, et al. Cavernous angioma: a review of 126 collected cases and 12 new clinical cases. Neurosurgery 18, 162-172 (1986).

11. Gelal F, F. H., Rezanko T, et al. Giant cavernous angioma of the temporal lobe: a case report and review of the literature. Acta Radiol 46, 310-313 (2005).

12. Khosla VK, B. A., Mathuriya SN, et al. Giant cystic cavernoma in a child. J Neurosurg 60, 1297-1299 (1984).

13. Kim YJ, K. J., Kim NR, Kim HS. Imaging findings of giant cavernous malformation with focal infiltrative pattern. Pediatr Radiol 37, 1039-1042 (2007).

14. Siddiqui AA, J. R. Neoplastic growth of cerebral cavernous malformation presenting with impending cerebral herniation: a case report and review of the literature on de novo growth of cavernomas. Surg Neurol 56, 42-45 (2001).

15. Kan P, T. M., Osborn A, Blaser S, Couldwell WT. Radiographic features of tumefactive giant cavernous angiomas. Acta Neurochir (Wien) 150, 49-55 (2007).

16. Del Curling O Jr, K. D. J., Elster AD, Craven TE. An analysis of the natural history of cavernous angiomas. J Neurosurg 75 702-708 (1991).

17. Anderson RC, C. E. J., Ozduman K, Laurans MS, et al. Clinicopathological review: giant intraventricular cavernous malformation. Neurosurgery 53, 374-378 (2003).

18. de Andrade GC, P. M., Braga FM. Giant cavernous angioma: report of two cases. Arq Neuropsiquiatr 60, 481-486 (2002).

19. Hayashi T, F. M., Shyojima K, et al. Giant cerebellar hemangioma in an infant. Childs Nerv Syst 1, 230-233 (1985). 